\title{
Research on Dynamic Performance of Coupler for Rescue EMUs
}

\author{
Fang Kai $^{1, a}$, Wu Pingbo ${ }^{1, b}$, Wang Qunsheng ${ }^{1, c}$ \\ ${ }^{1}$ State Key Laboratory of Traction Power, Southwest Jiaotong University, Chengdu, China 610031 \\ afangkai0975@163.com, bvehicle@home.swjtu.edu.cn, '862121202@qq.com
}

Keywords: rescue multiple units; coupler draft gear; dynamic performance; simulation

\begin{abstract}
In order to study the influence of train running parameters to the rescue electric multiple units (EMUs) coupler dynamic performance, a train-coupler system simulation model was established. The influence of different braking level, braking time and braking original speed to the longitudinal force and nodding angle of coupler during different types of multiple units after rescue coupled braking was researched. The result shows that the braking acceleration, braking time and vehicle grouping mode would have an influence on the dynamic performance of the coupler signally.
\end{abstract}

\section{Introduction}

At present, the electric multiple units (EMUs) operated in China Railway are CRH1, CRH2, CRH3, CRH5 and CRH380 series, etc. Each different series of EMUs can't be coupled with each other due to different types and height of coupler installation. This causes different types of EMUs can't achieve mutual rescue and can only use the locomotive coupling EMUs to achieve rescue $[1,2]$. In the process of EMUs running, the coupler force increases sharply and coupler performance becomes worse because of the train longitudinal impact caused by the factors such as traction and braking. Two types of domestic EMUs (EMUs A and EMUs B) with different coupler installation height conducted the rescue test by using the transition coupler and it showed that coupler draft gear, anti-yaw damper and car body appeared different degree of deformation after applying emergency braking. This article established a train-coupler system model and mainly analyzed under the braking condition, the influence of running parameter to the coupler dynamic performance after the EMUs rescue coupled.

\section{The dynamical model of the train}

This article considered the relationship between the internal structure of coupler draft gear and the movement in detail and established the multi-body dynamics model of train. Considering the real motion and stress condition, the model of coupler in every car end is considered into two rigid bodies including coupler yoke and middle coupler part. The whole coupler graft gear has four rigid bodies after two cars coupled. Considering the relative motion between the middle couplers is negligible as it is very small during the actual running, the two middle couplers are considered as a whole for simplification of the model, as Fig. 1 shown. Coupler yoke has three degrees as longitudinal extension, respectively movement and nodded. The middle coupler with a nod degree of freedom can rotate around the cross pin.

For the coupler graft gear of every car, create four force elements in the multi-body system dynamics software SIMPACK including one force element of coupler draft gear, two force elements of vertical spring located in front and back beam of coupler yoke respectively and one force element of between coupler beam and car body. The coupler dynamic model is shown in Fig. 2. 


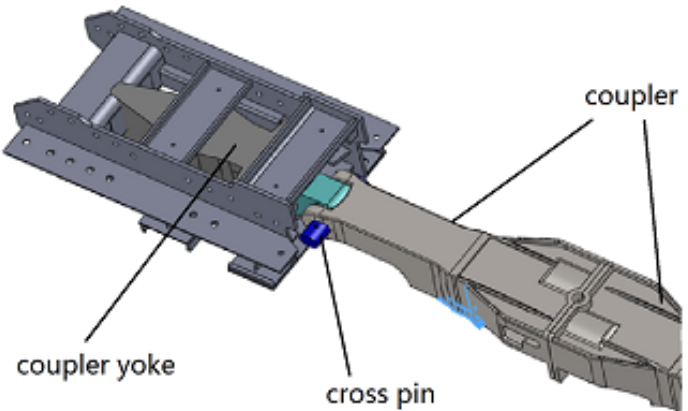

Fig.1 3D model of coupler system

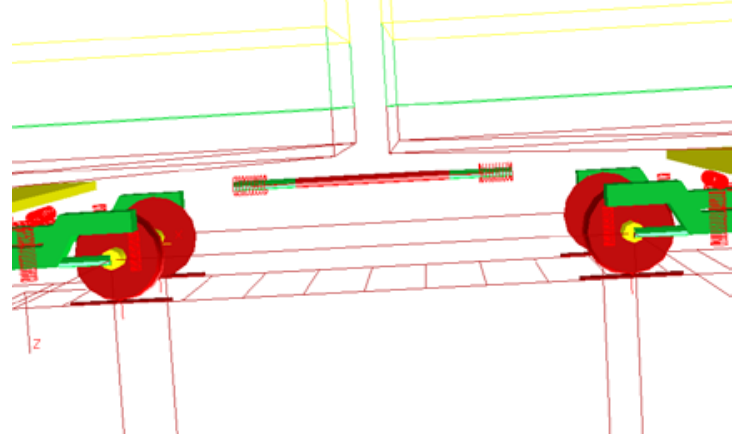

Fig.2 Dynamic model of coupler system

Our main research purpose is to study the dynamic performance of the coupler in the rescue car coupled to the car need to be rescued. It has certain difficulty to establish a dynamic simulation model which EMU B with 16 cars rescue EMU A with 16 cars (16B-16A). We made some simplification of the 16B-16A model in order to ensure the feasibility and accuracy of the simulation analysis and then created the new 16B-16A model which was composed of 5 cars and 2 mass with longitudinal degree of freedom. The train dynamic model is shown in Fig. 3. The non-linear wheel/ rail geometric interaction, wheel/ rail force and suspension parameter are concerned in the process of modeling.

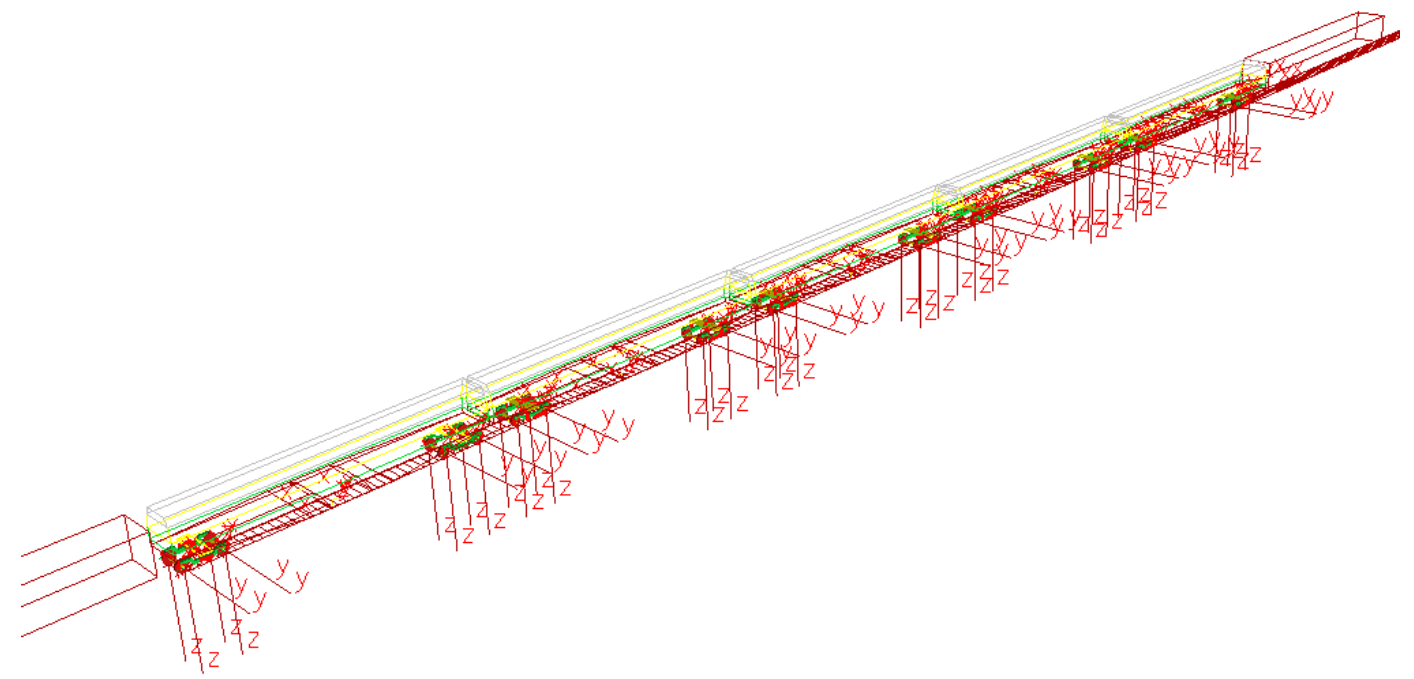

Fig.3 Multi-body dynamic model of the train

\section{Validation of the train dynamic model}

In order to verify the accuracy of the train dynamic model, we compared the coupler force measured in simulation with the coupler measured in experiment test. In the line test condition which the EMU B with 16 cars rescue coupled EMU A with 16 cars and EMU B take emergency braking at the speed of $20 \mathrm{~km} / \mathrm{h}$, measured the coupler force changing with the braking time. In the simulation analysis, use the braking parameter as shown in Tab. 1.

Tab. 1 braking parameter of every vehicle

\begin{tabular}{|c|c|c|c|}
\hline Parameter & Abbreviation & Magnitude & Unit \\
\hline Total weight & Mc & 45 & $\mathrm{t} / \mathrm{s}^{2}$ \\
\hline Braking deceleration & $\mathrm{a}$ & -1.3949 & $\mathrm{kN}$ \\
\hline Braking force & Fx & -62.77 & $\mathrm{~m}$ \\
\hline $\begin{array}{c}\text { Distance to the mass } \\
\text { center of car body }\end{array}$ & D & 1.09 & $\mathrm{kN} . \mathrm{m}$ \\
\hline $\begin{array}{c}\text { Nod torque caused by } \\
\text { braking }\end{array}$ & Tc & -68.42 & \\
\hline
\end{tabular}




\begin{tabular}{|c|c|c|c|}
\hline $\begin{array}{c}\text { Rotational inertia of } \\
\text { car body nodding }\end{array}$ & $\mathrm{J}$ & 1654534 & $\mathrm{~kg} \cdot \mathrm{m}^{2}$ \\
\hline
\end{tabular}

By comparing the coupler force measured in line test (shown as Fig. 4a) and in simulation analysis (shown as Fig. 4b), it shows that the maximum coupler force in test is $735.6 \mathrm{kN}$ and the maximum coupler force in simulation is $705.4 \mathrm{kN}$, and the difference is $30.2 \mathrm{kN}$. In addition, the change trend of coupler force in line test and simulation results are basically the same and the simulation results can generally represent the variation of coupler force in line test. So it illustrates the train-coupler system model is feasible for studying the influence of train running parameter to the coupler force and can accurately reflect the coupler dynamic performance.

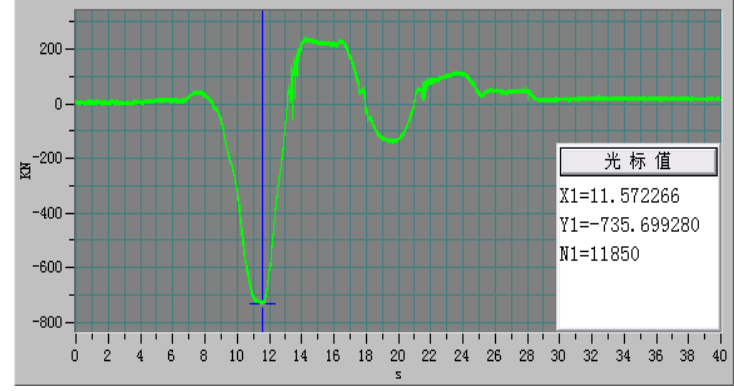

(a) the coupler force measured in line test

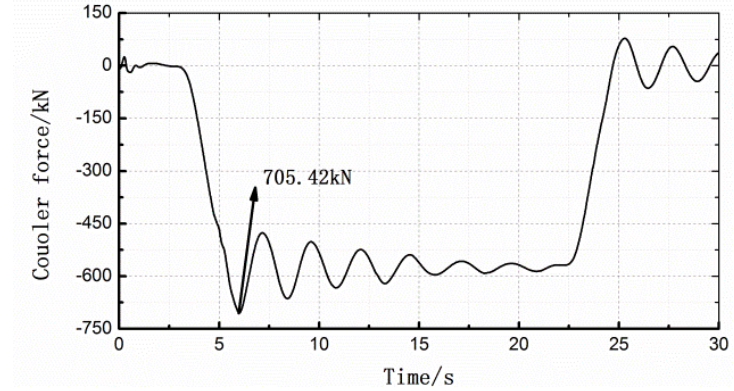

(b) the coupler force of simulation analysis

Fig. 4 Comparison of field test and simulation result

\section{The influence of train running parameter to the coupler dynamic performance}

When studying the influence of train running parameter to the coupler dynamic performance, the train runs on a straight line and there is no track irregularity. The main influence of parameters on the coupler dynamic performance is the longitudinal coupler force and the coupler rotation angle.

\subsection{Braking acceleration}

According to the magnitude of the acceleration, the braking mode of the high-speed EMU is divided into emergency braking and service braking (level $1 \mathrm{~N}$ to $7 \mathrm{~N}$ ). The running speed of the train in this section is $60 \mathrm{~km} / \mathrm{h}$ and the braking time is $2.5 \mathrm{~s}$, five different braking level (shown in Tab. 2) is considered and the simulation results is shown as Fig. 5a and Fig. 5b.

Tab. 2 five braking level

\begin{tabular}{|c|c|c|c|}
\hline Braking Grade & $60 \mathrm{~km} / \mathrm{h}$ & $80 \mathrm{~km} / \mathrm{h}$ & $120 \mathrm{~km} / \mathrm{h}$ \\
\hline Emergency Braking & $1.3949 \mathrm{~m} / \mathrm{s}^{2}$ & $1.3949 \mathrm{~m} / \mathrm{s}^{2}$ & $1.3949 \mathrm{~m} / \mathrm{s}^{2}$ \\
\hline 7N Braking & $0.7472 \mathrm{~m} / \mathrm{s}^{2}$ & $0.7206 \mathrm{~m} / \mathrm{s}^{2}$ & $0.6167 \mathrm{~m} / \mathrm{s}^{2}$ \\
\hline 6N Braking & $0.6500 \mathrm{~m} / \mathrm{s}^{2}$ & $0.6268 \mathrm{~m} / \mathrm{s}^{2}$ & $0.5364 \mathrm{~m} / \mathrm{s}^{2}$ \\
\hline 5N Braking & $0.5528 \mathrm{~m} / \mathrm{s}^{2}$ & $0.5331 \mathrm{~m} / \mathrm{s}^{2}$ & $0.4563 \mathrm{~m} / \mathrm{s}^{2}$ \\
\hline $4 \mathrm{~N}$ Braking & $0.4583 \mathrm{~m} / \mathrm{s}^{2}$ & $0.4415 \mathrm{~m} / \mathrm{s}^{2}$ & $0.3761 \mathrm{~m} / \mathrm{s}^{2}$ \\
\hline
\end{tabular}

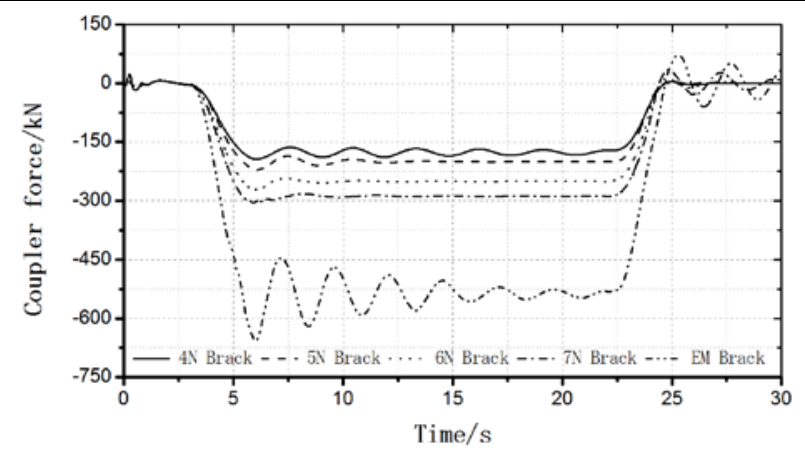

(a) Influence on coupler force

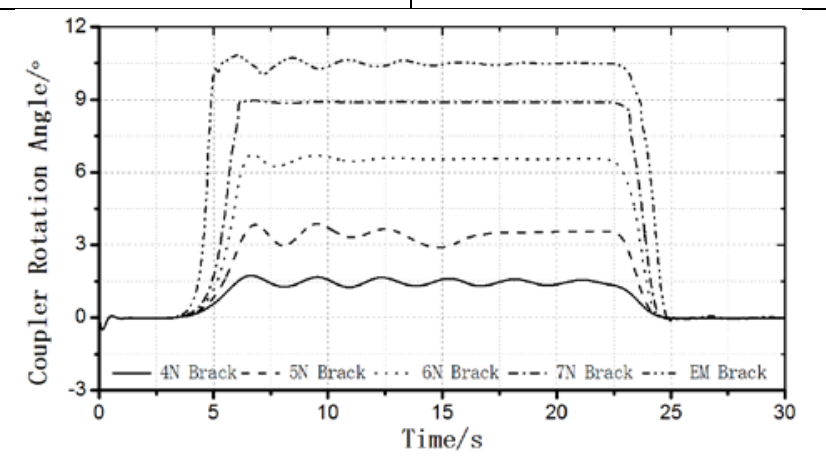

(b) Influence on coupler rotation angle

Fig. 5 Influence of different braking level on coupler dynamics

It can be seen from the results that different braking level has different breaking acceleration, and the coupler force reduces signally with the decrease of the acceleration, as shown in Fig.5a.This is because the decrease of the braking acceleration led to decrease of the longitudinal impact between 
the two rescue coupled EMUs. Seen from the curve of the emergency braking, in the process of coupler force reaches the maximum to return to the balance, there are some waves. This is because the coupler draft gear can't absorb the impact energy fully at once and the waves reduce until the balance with the decrease of the longitudinal impact energy. Seen from Fig. 5b, the maximum rotation angle of coupler also increases with the increase of the braking acceleration. There are also some waves in the process of coupler rotation angle reaches the maximum to return to the balance and the reason is also the coupler graft gear can’t absorb the impact energy fully at once.

\subsection{Braking time}

Braking time means the time deceleration increase from zero to a specified magnitude costs when the train braking. In this section, the train running speed is $60 \mathrm{~km} / \mathrm{h}$ and the braking level is $7 \mathrm{~N}$. Five different level of braking time from $2 \mathrm{~s}$ to $5 \mathrm{~s}$ is chosen and the simulation results are shown as Fig. 6a and Fig. 6b.

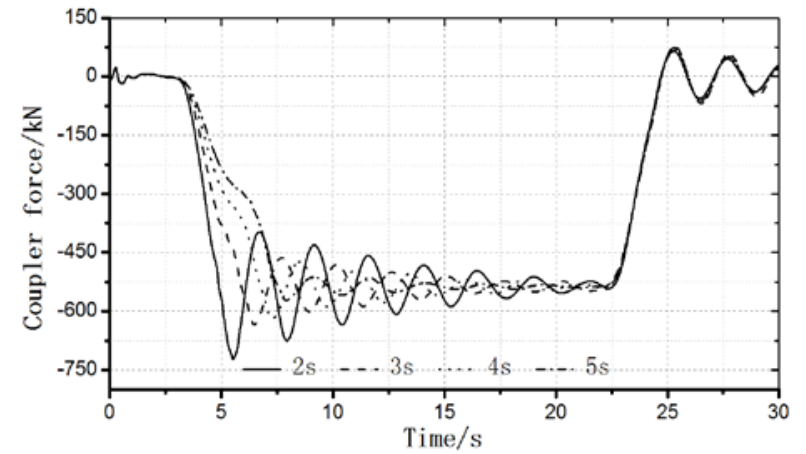

(a) Influence on coupler force

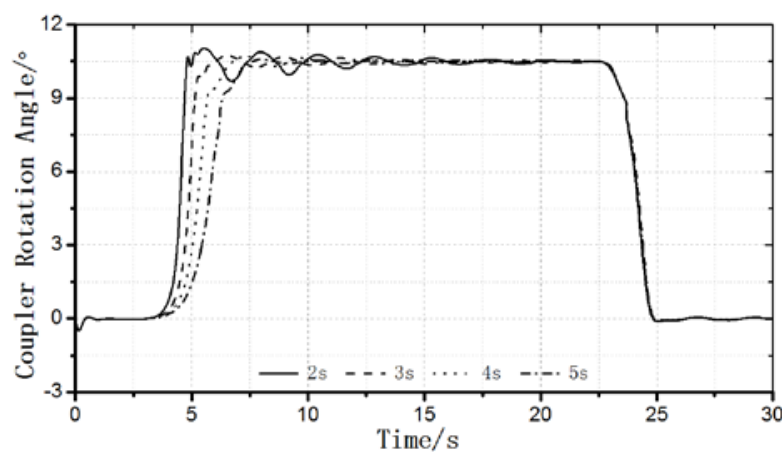

(b) Influence on coupler rotation angle

Fig. 6 Influence of different braking time on coupler dynamics

It can be seen from the results that different braking time will also influence the coupler force and the coupler rotation angle. When the braking time is shortest for $2 \mathrm{~s}$, the coupler is the largest for $722.4 \mathrm{kN}$. The shorter the braking time is, the greater the coupler force will be and the wave of coupler force from the maximum to the balance is greater. The cause of this phenomenon is when the braking time becomes shorter, the change of the acceleration will be greater and the longitudinal impact energy between EMUs A and EMUs B will become greater.

\subsection{Running speed}

When the train breaks in different speed, the breaking acceleration is the same. This section studies the coupler dynamic performance when the train taking emergency braking in different running speed. The running speed of the train is respectively $60,80,100$ and $120 \mathrm{~km} / \mathrm{h}$ and the simulation results are shown as Fig.7a and Fig. 7b.

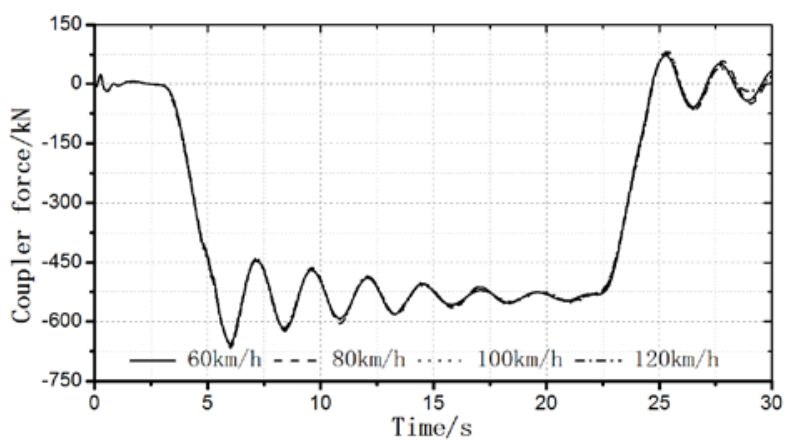

(a) Influence on coupler force

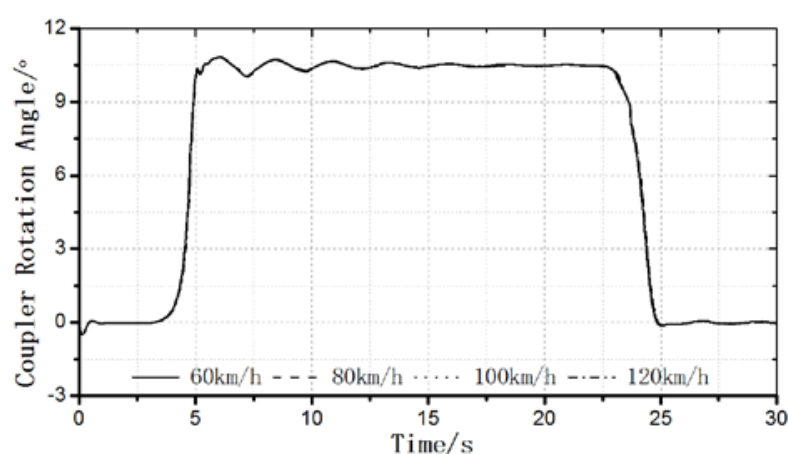

(b) Influence on coupler rotation angle

Fig. 7 Influence of different running speed on coupler dynamics

It can be seen from the results that the magnitude and the change trend of the coupler force and coupler rotation angle are basically the same although the train braking in different running speed. It is because in the original state, vehicles running synchronously and have the same original speed. The coupler force in the braking moment depends on the braking acceleration and has nothing to do with the original running speed. 


\subsection{Vehicle grouping}

The simulation model used above is the 16B-16A rescue coupling model. This section studies the coupler dynamic performance under the condition that the running speed is $60 \mathrm{~km} / \mathrm{h}$ when the train taking emergency braking with four different vehicle grouping modes. And the four grouping mode is 16B-16A, 16B-8A, 8B-16A and 8B-8A. Simulation results are shown as Fig. 8a and Fig. 8b.

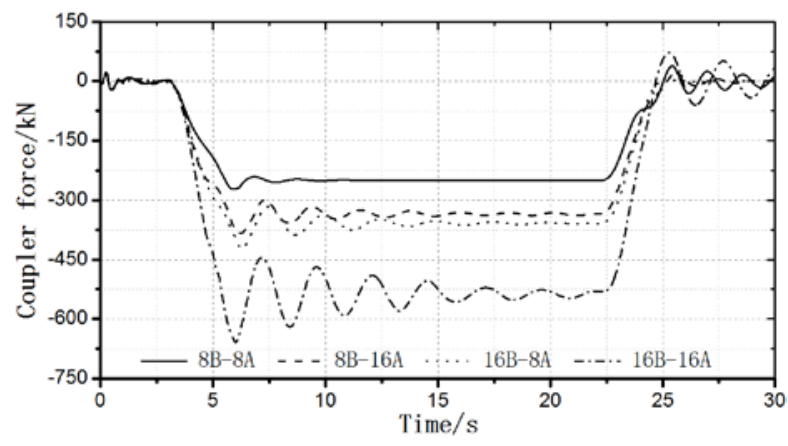

(a) Influence on coupler force

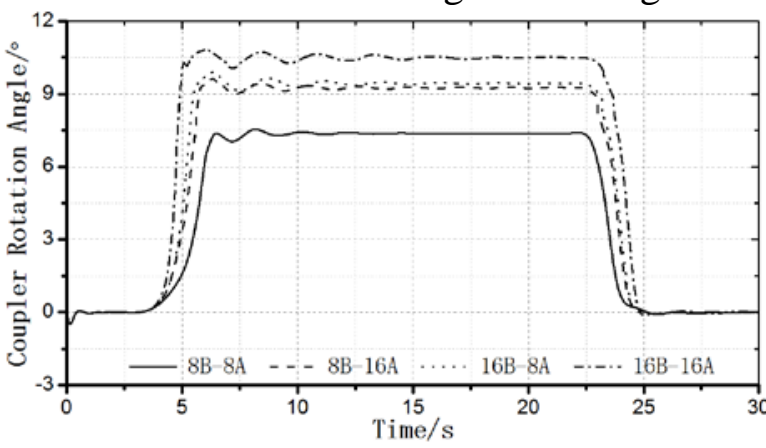

(b) Influence on coupler rotation angle

Fig. 8 Influence of different grouping mode on coupler dynamics

It can be seen from the results that different grouping mode will influence the coupler force and the coupler rotation angle greatly. When the vehicle grouping mode is $16 \mathrm{~B}-16 \mathrm{~A}$, the coupler force has the maximum of $660.6 \mathrm{kN}$ and the coupler rotation angle has the maximum of $10.9^{\circ}$. Long grouping train has the larger coupler force and coupler rotation angle than short grouping train. This is because longer grouping train has greater quality, and the greater quality will cause greater longitudinal impact energy when the train braking.

\section{Conclusions}

This article developed the dynamic simulation model of two different type EMU rescue coupled and verified the feasibility and accuracy of the model by comparing with the line test data. Through the simulation analysis, have the following conclusion:

(1) This article developed the relatively perfect dynamic model of coupler draft gear according to the coupler force curve measured in line test and the coupler model. Based on the coupler dynamic model, developed the multi-grouping vehicle dynamic model and reproduced the variation rule of the coupler force measured in line test.

(2) Braking acceleration, braking time and vehicle formation form will influence dynamic performance of the coupler signally. With the increase of braking acceleration, the longitudinal impact of the vehicle significantly enhanced, resulting in coupler force and coupler nodding angle have increasing trend obviously. With the decrease of the braking time, longitudinal coupler force increase gradually. The train with longer grouping will lead to the increase of longitudinal couple force and coupler rotation angle obviously.

(3) The running speed of the train has little influence on the dynamic performance of the coupler. Vehicles run synchronously at the same original speed and the coupler force is independent with the original braking speed.

\section{Acknowledgement}

This research was financially supported by the National 863 plan [2012AA112001], the National Natural Science Fund of China [U1334206], and the National 973 plan [2011CB711106].

\section{References}

[1] Li Rui-chun, Unified Design for Draught-gear for Electric Multiple Unit [J], Urban Mass Transit.2013, (8):64-69.

[2] Gao Jun,etc. Discussion of the Standard for Transition Couplers on High Speed Multiple Units 
[J], Rolling Stock.2013, 53(8):25-27.

[3] Thomas Geike, Understanding High Coupler Forces at Metro Vehicles [J], Vehicle System Dynamics.2007, 45(4):389-396.

[4] Yuan Yao, etc. The Stability Mechanism and its Application to Heavy-haul Couplers with Arc Surface Contact [J], Vehicle System Dynamics. 2013,51(9):1324-1341.

[5] Chen Kai, etc. Test and Research on Mutual Rescue between Multiple Units of Different Types [J], Rolling Stock.2014,52(5):1-5.

[6] R.C. Li, Research on high-speed train and raising speed train vehicle hook buffer mechanism[J], Railw. Locomotive Car 24 (2004), pp. 15-21.

[7] Ma Wei-hua, etc. Coupler dynamic performance analysis of heavy haul locomotives[J]. Vehicle System Dynamics. 2012, 50(9): 1435-1452

[8] Xu Zi-qiang, etc. Analysis of coupler rotation angle in heavy haul locomotives[J]. Proc IMechE, Part F:J. Rail Rapid Transit.2013,(4):1-10

[9] Ma W, Luo S, Song R. Coupler dynamic performance analysis of heavy haul locomotives[J]. Veh Syst Dyn. 2012;50(9):1435-1452. 